\title{
Estudo da deformação permanente de britas granito- gnaisse para uso em base e sub-base de pavimentos
}

\author{
Caroline Dias Amancio de Lima ${ }^{1}$, Laura Maria Goretti da Motta ${ }^{2}$, Antônio Carlos Rodrigues \\ Guimarães $^{3}$
}

1PEC-COPPE, Universidade Federal do Rio de Janeiro, Rio de Janeiro, RJ, Brasil, engcarolinelima@gmail.com 2PEC-COPPE, Universidade Federal do Rio de Janeiro, Rio de Janeiro, RJ, Brasil, laura@coc.ufrj.br 3Instituto Militar de Engenharia, Rio de Janeiro, RJ, Brasil, guimaraes@ime.eb.br

\section{Recebido:}

06 de outubro 2016

Aceito para publicação:

19 de abril 2017

Publicado:

31 de agosto de 2017

Editor de área:

Jorge Barbosa Soares, UFC

\section{Palavras-chaves:}

Mecânica dos pavimentos.

Deformação permanente.

Brita graduada.

Material de pavimentação.

\section{Keywords:}

Pavement Mechanics.

Permanent deformation.

Crushed stone.

Pavement material.

DOI:10.14295/transportes.v25i2.1262

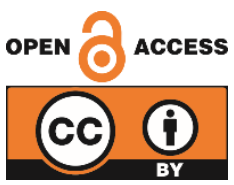

\begin{abstract}
Materials selection for pavement construction is very important because they influence the pavement performance. Two different materials with similar characteristics were studied for different grain size distribution curves, same for both. The granitegneiss crushed stones analyzed in this study were employed in base and sub-base of real pavements of the Rio de Janeiro city. The permanent deformation was evaluated here by results obtained from tests performed in repeated load triaxial equipment. Nine different stress stages were applied per curve, different test for each stress stage and at least 150,000 cycles of loading. The results allowed permanent deformation analysis, the shakedown occurrence, resilient modulus analysis, influence of particle size distribution, correlation of the results, and obtain new parameters of Guimarães model (2009).
\end{abstract}

\begin{abstract}
RESUMO
A escolha do material para construção do pavimento tem muita importância porque influencia diretamente no seu desempenho. Neste trabalho, foram estudadas diferentes curvas granulométricas aplicadas para o mesmo material e a outro material distinto com algumas características semelhantes. As britas graduadas do tipo granito-gnaisse analisadas neste estudo foram aplicadas em base e sub-base de pavimentos reais da cidade do Rio de Janeiro. A deformação permanente, defeito estrutural indesejado, foi avaliada aqui por meio de resultados obtidos de ensaios realizados no equipamento triaxial de cargas repetidas. Foram utilizados nove pares de tensões distintos por curva, sendo aplicado um par de tensões e no mínimo 150 mil ciclos de carregamentos por corpo de prova. Os resultados possibilitaram análises da deformação permanente, ocorrência do shakedown, módulo de resiliência, influência granulométrica, e correlação entre os resultados, bem como a inserção de novos parâmetros no banco de dados do modelo de Guimarães (2009).
\end{abstract}

\section{INTRODUCTION}

0 pavimento é uma estrutura de múltiplas camadas finitas destinadas a resistir às intempéries e solicitações de cargas dos veículos. No Brasil, a grande extensão tanto da malha pavimentada como da não pavimentada e o uso intenso de materiais pétreos e solos na construção e manutenção dos pavimentos rodoviários justificam as pesquisas realizadas em busca de entendimento e previsão do comportamento dos pavimentos rodoviários e seus componentes.

Um dos defeitos resultantes da passagem de veículos é o Afundamento de Trilha de Roda (ATR), um tipo de defeito estrutural, que pode ser derivado da contribuição acumulativa da deformação permanente de uma ou mais camadas do pavimento e do subleito, e quando excessiva ou brusca resulta em ruptura plástica, sendo um dos principais mecanismos de ruptura de pavimentos. 
Segundo Marangon e Motta (2006), a natureza do carregamento cíclico na estrutura, cria a necessidade de se verificar a deformação permanente após um determinado número de aplicações de carga no sentido de determinar se tais deformações permanentes podem conduzir à ruptura ou se tende à estabilização, questão particular de obras de pavimentação. De acordo com Guimarães (2009), devem-se buscar solos granulares e finos que apresentem acomodamento das deformações permanentes com o número de ciclos de solicitações, fenômeno conhecido como shakedown, para ter a garantia de que não haverá ruptura plástica.

Junto com a estimativa da deformação permanente está o entendimento do seu surgimento para poder dimensionar o pavimento de forma mais racional do que vem sendo dimensionado. Para tanto, é necessária a avaliação da influência de alguns fatores, tais como: variação do estado de tensões, variação de umidade na compactação, variação do módulo de resiliência, variação da distribuição e granulometria dos materiais, etc. Essas análises foram realizadas neste estudo para identificar as qualidades e melhor aproveitar os materiais pétreos.

Optou-se pelo estudo de brita graduada simples, visto que é muito utilizada no país como base e sub-base de pavimentos asfálticos. Selecionaram-se duas britas granito-gnaisse, de pedreiras distintas, comumente aplicadas em camadas dos pavimentos no estado do Rio de Janeiro, com o objetivo de compreender melhor a deformação permanente deste tipo de material.

\section{DEFORMAÇÃO PERMANENTE}

A deformação permanente ou deformação plástica é a parcela da deformação total que não retorna à sua posição original. Esses deslocamentos são cumulativos e não recuperáveis ao longo da vida do pavimento. A deformação permanente vista na superfície do pavimento é o resultado de todos eles por toda a estrutura do pavimento como Barksdale (1972) expôs. Para ele, a deformação permanente, ou afundamentos de trilha de roda, é oriunda da contribuição de todas as camadas como mostrado na Equação (1).

$$
\delta_{\text {total }}^{p}=\sum_{i=1}^{n} \varepsilon_{p}^{i} h_{i}
$$

Onde,

$\delta_{\text {total }}^{\mathrm{p}}$ : profundidade total do afundamento;

$\varepsilon_{\mathrm{p}}^{\mathrm{i}}$ : deformação específica plástica média da i-ésima camada;

$\mathrm{h}_{\mathrm{i}}$ : espessura da i-ésima camada;

n: número total de camadas.

0 estudo da deformação permanente fornece informações precisas para melhor aproveitamento das qualidades dos materiais disponíveis, auxiliando no dimensionamento racional de pavimentos. Frente a isso, alguns países, como a Inglaterra, passaram a utilizar na década de 1990 métodos de ensaios testando diferentes modelos até encontrar um modelo adequado de previsão para as deformações permanentes dos materiais locais. Também foi proposta uma classificação dos resultados em três categorias: acomodamento (A), deformação permanente crescente a pequenas taxas (B) e colapso (C). Seguindo a linha dos ingleses, Guimarães (2009) propôs um modelo para os materiais nacionais e introduziu o comportamento $\mathrm{AB}$ encontrado para materiais tropicais brasileiros, em especial solo de comportamento laterítico e lateritas. 0 modelo de Guimarães (Equação 2) foi o modelo de previsão escolhido para análise dos materiais aqui estudados. 


$$
\varepsilon_{p}(\%)=\psi_{1} \cdot\left(\frac{\sigma_{3}}{\rho_{0}}\right)^{\psi_{2}} \cdot\left(\frac{\sigma_{d}}{\rho_{0}}\right)^{\psi_{3}} \cdot N^{\psi_{4}}
$$

Onde,

$\varepsilon_{\mathrm{p}}(\%):$ deformação permanente específica;

$\Psi_{1}, \Psi_{2}, \Psi_{3}, \Psi_{4}$ : parâmetros de regressão (parâmetros de deformabilidade permanente);

$\sigma_{3}$ : tensão confinante;

$\sigma_{\mathrm{d}}$ : tensão desvio;

$\rho_{0}$ : tensão de referência (tensão atmosférica);

$\mathrm{N}$ : número de ciclos de aplicação de carga.

Vale salientar que as posições dos agregados devido à proporção e aos diferentes tamanhos podem estar intimamente relacionados ao comportamento da camada do pavimento, como também a umidade, o índice de vazios, a frequência, o estado de tensões, etc. Esses fatores devem ser levados em conta além da qualidade intrínseca do material verificado pelos ensaios convencionais como Abrasão Los Angeles e durabilidade, e outros índices como de forma.

A Brita Graduada Simples (BGS), material selecionado para estudo, é um dos materiais com poucos parâmetros de deformabilidade permanente determinados no país, embora seja muito utilizada como base e sub-base de pavimentos asfálticos, em diferentes composições de faixas granulométricas. Dificilmente realizam-se ensaios de deformação permanente no equipamento triaxial de cargas repetidas para esse material, visto que ainda hoje é utilizado o ensaio CBR para o dimensionamento de pavimentos. Os órgãos rodoviários têm especificações que prescrevem a distribuição granulométrica por meio de faixas e critérios clássicos de qualidade dos agregados para determinada aplicação.

Lima e Motta (2015a) apresentaram uma curva de BGS montada utilizando os parâmetros do método Bailey, utilizado para misturas asfálticas, e concluíram que a composição granulométrica teve considerável influência na deformação permanente total do material. A pesquisa sugeriu a necessidade de se estabelecer parâmetros de composição granulométrica associados ao método Bailey aplicado à análise de materiais de base e sub-base ou aos critérios de empacotamento do esqueleto mineral.

Para analisar a influência granulométrica e como o parâmetro índice de vazios auxilia na previsão de deformações permanentes, foi composta mais uma curva, baseada no método de Fuller-Talbot com $n=0,45$. Após os ensaios de deformação permanente, os corpos de provas foram destorroados e foi realizada a granulometria de cada um deles mostrando que houve variação que pode ter sido causada por quebra ou abrasão. Lima e Motta (2015b) concluíram que a distribuição granulométrica exercia influência nos resultados obtidos no equipamento triaxial de cargas repetidas, e que o índice de vazios apresentava um leve padrão: quanto maior a quantidade de vazios, maior a deformação permanente resultante.

Posteriormente, Lima e Motta (2016) incluíram mais um material na análise para verificar a consistência do que foi constatado quanto à dependência da distribuição granulométrica. Com novos parâmetros do modelo de Guimarães (2009), mais uma vez constataram a importância de se realizar ensaio de deformação permanente, e que a granulometria adotada influenciava. Quanto às tensões, em relação aos parâmetros de deformabilidade do modelo de Guimarães (2009), para brita graduada foram: o parâmetro $\psi_{2}$ referente à tensão confinante com valornegativo indica que a tensão confinante é inversamente proporcional ao aumento de 
deformações permanentes, e o parâmetro $\psi_{3}$ referente a tensão desvio, é o que exerce maior influência no aumento das deformações permanentes acumuladas.

Os trabalhos mencionados, assim como este e outros nacionais e internacionais, fizeram estudo da deformação permanente baseada na Teoria do Shakedown. Essa teoria, segundo Cerni et al. (2012), comporta a ideia de estabelecer um nível de tensão limite no qual o pavimento passa a ser estável com o surgimento de tensões residuais e o fim do acréscimo de deformações permanentes. Para Werkmeister et al. (2004) quando a taxa de acréscimo de deformação permanente atinge $10^{-7} \mathrm{~mm} /$ ciclo de carga já se pode considerar a ocorrência do fenômeno do shakedown.

A proposição gráfica de Dawson e Wellner (1999) e Werkmeister (2003) permite a classificação de três comportamentos (A, B, e C), representados na Figura 1, que foram utilizados para interpretação dos resultados dos ensaios e na identificação dos níveis de comportamento do material. O comportamento AB proposto por Guimarães (2009) para materiais brasileiros também está apresentado nesta figura. Observe-se que os dois eixos do gráfico devem estar em escala logarítmica, no eixo horizontal está representada a deformação permanente acumulada e no eixo vertical está a taxa de acréscimo da deformação permanente por ciclo de carga, em ordem decrescente.

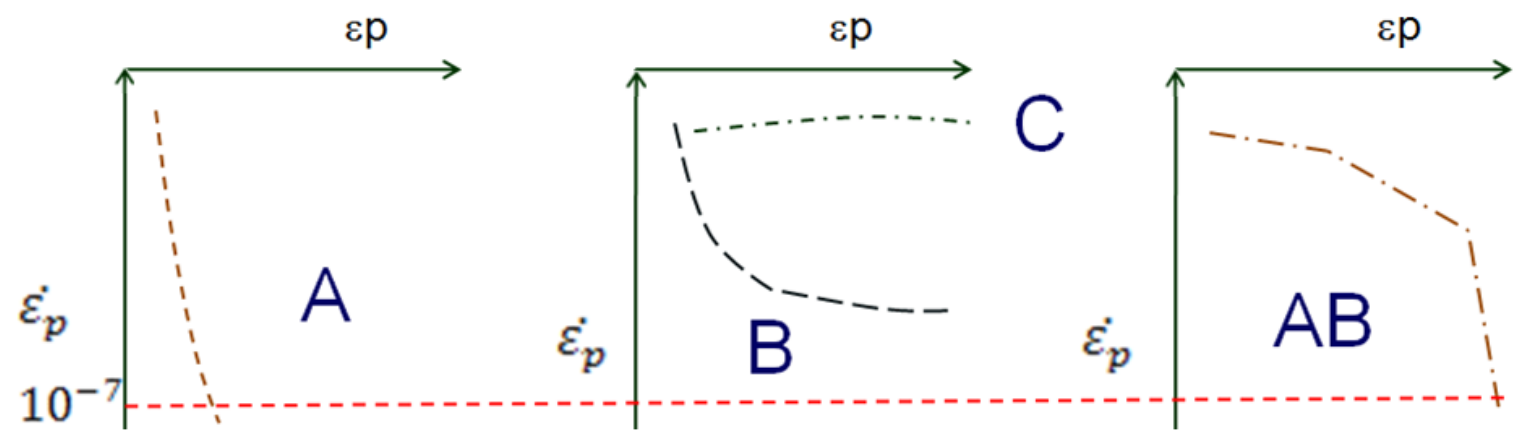

Figura 1. Níveis de comportamento da proposição de Dawson e Wellner (1999) e Guimarães (2009) para estudo do shakedown (Lima e Motta, 2016)

O comportamento de nível A (acomodamento plástico ou Shakedown) apresenta apenas resposta elástica após finitas aplicações de ciclos de carga e pequena deformação permanente acumulada. 0 comportamento de nível B apresenta uma elevada taxa de acréscimo de deformação permanente nos primeiros ciclos iniciais e tende a um posterior acomodamento. Já o nível C (colapso) apresenta altas taxas de acréscimo de deformação permanente e sucessivos incrementos de deformação permanente a cada ciclo podendo o material chegar à ruptura. No nível $\mathrm{AB}$ há significativas deformações iniciais seguido de acomodamento plástico.

\section{MATERIAIS E MÉTODOS}

Os materiais estudados foram duas britas graduadas de granito-gnaisse provenientes do estado do Rio de Janeiro, de duas pedreiras, que comumente são aplicadas em obras de pavimentação na capital do estado. As BGS estudadas foram denominadas: material 1, BGS utilizada em alguns trechos na obra da via TransCarioca, e material 2, BGS da pedreira Petra Agregados.

0 trabalho experimental resultou na caracterização do material e o total de 54 ensaios de deformação permanente e 72 ensaios de módulo de resiliência no equipamento triaxial de cargas repetidas para análise de 6 curvas granulométricas. A preparação dos corpos de prova foi feita na energia Proctor modificada. 
As três diferentes distribuições granulométricas trabalhadas com as duas BGS foram construídas em laboratório baseadas em três métodos distintos: método da curva de Fuller-Talbot, que propõe a graduação ideal pela densidade máxima, método Bailey para curva de comportamento fino, desenvolvido para seleção do esqueleto mineral para misturas asfálticas considerando o intertravamento, e método de Alfred, modelo de empacotamento utilizado para cerâmica e concreto, e que leva em consideração o diâmetro mínimo do material. Foram aqui denominadas como C2, C3 e C4 para o material 1 e C5, C6, e C7 para o material 2, correspondentes à ordem dos métodos citados. As curvas analisadas estão apresentadas na Figura 2, enquadradas nas faixas da norma ET-DE-P00/008 do DER/SP.

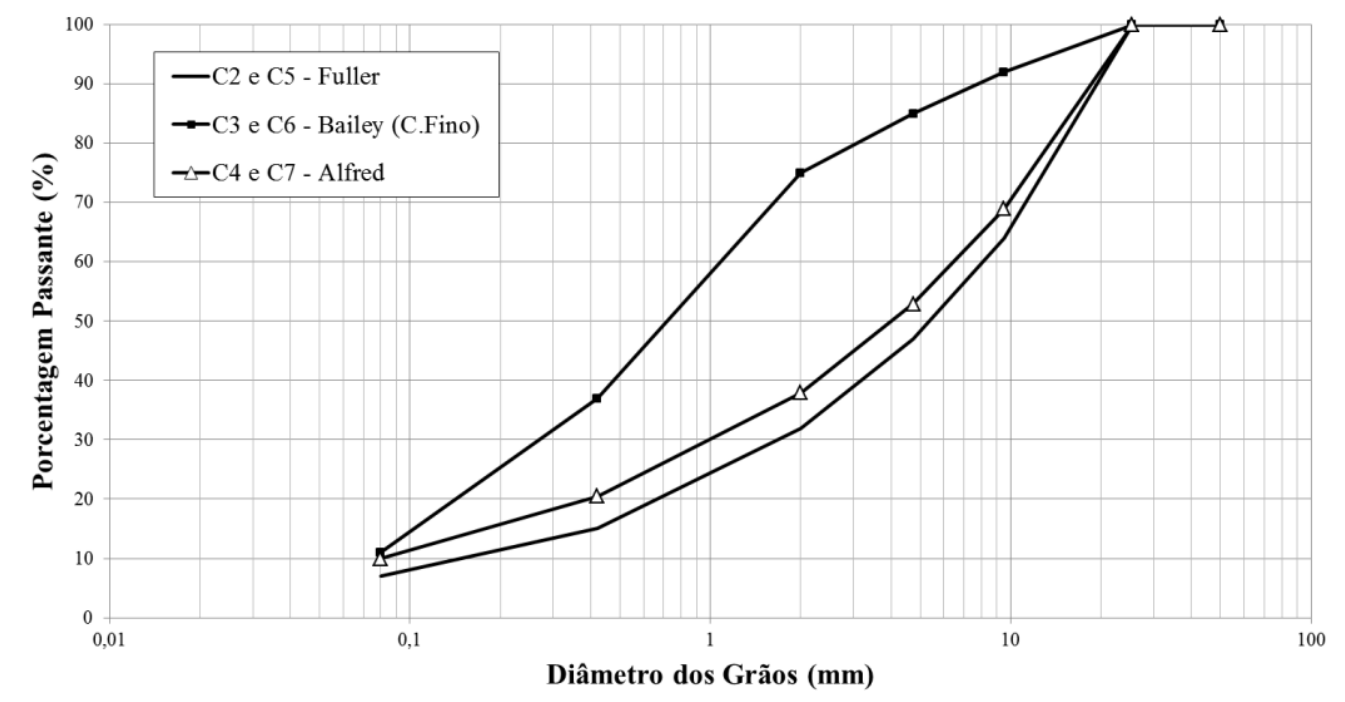

Figura 2. Representação das curvas granulométricas das duas britas graduadas de granito-gnaisse deste estudo

Para caracterização dos materiais foram seguidas as normas apresentadas na Tabela 1. Os limites foram baseados na norma ET-DE-P00/008 do DER/SP, utilizada para construção de alguns trechos da via citada. 0 resultado do Slake Durability Test, com o qual é possível medir a estabilidade e durabilidade de solo e rochas brandas, é do tipo I para ambos os materiais indicando que é um excelente material quanto ao desgaste em meio úmido.

Tabela 1: Caracterização física das BGS utilizadas nesta pesquisa

\begin{tabular}{lccc}
\hline \multicolumn{1}{c}{ Propriedades/Parâmetros } & Material 1 & Material 2 & ET-DE-P00/008 DER-SP \\
\hline Massa específica aparente - Agr. Graúdo (DNER-ME 195-97) & 2,62 & 2,64 & - \\
Massa específica aparente - Agr. Fino (DNER-ME 084-95) & 2,70 & 2,60 & - \\
Absorção (DNER-ME 081-98) & 0,80 & 0,53 & - \\
Angularidade - Agr. Graúdo (AASHTO T326-05) & 45,9 & 45,1 & - \\
Angularidade - Agr. Fino (AASHTO T304-96) & 46,7 & 44,7 & - \\
Equivalente de areia (DNER-ME 054-97) & 68 & 71 & $>55 \%$ \\
Abrasão Los Angeles (DNER-ME 035-98) & 41 & 43 & $<50 \%$ \\
Slake Durability Test (ASTM D4644-08) & 99,52 & 99,36 & - \\
Treton (DNER-ME 399-99) & 24 & 22 & $\leq 60 \%$ \\
\hline
\end{tabular}

No equipamento triaxial de cargas repetidas, o número $\mathrm{N}$ de ciclos de carga adotado foi superior a 150 mil ciclos, desconsiderando o $1^{\circ}$ golpe para análise, e para cada curva granulométrica foram realizados 9 ensaios de Deformação Permanente (DP), cada um correspondente a um par de tensões, e 12 ensaios de Módulo de Resiliência (MR), sendo 9 MR realizados após os 
9 ensaios de DP e 3 MR realizados em corpos de prova distintos preparados especialmente para este ensaio. Os MR e as DP foram ensaiados à frequência de $1 \mathrm{~Hz}$ com exceção dos ensaios de DP para C6 e C7 realizados com frequência de $2 \mathrm{~Hz}$.

Os nove pares de tensões para análise da deformação permanente foram os mesmos utilizados nos trabalhos de Lima e Motta (2015a, 2015b, 2016) que foram baseados em Guimarães (2009) e no Manual de Execução de Trechos Monitorados da Rede Temática de Asfalto (2010). Para realização dos módulos de resiliência foram aplicadas as tensões normatizadas pelo DNIT 134/2010- ME.

\section{RESULTADOS E ANÁLISES}

Para as análises de módulo de resiliência (Tabela 2) optou-se por representar o seu valor em função da tensão confinante, de melhor enquadramento dentre os modelos simples e também pelo modelo composto. Os resultados apresentados na Tabela 2 são referentes aos três ensaios realizados para cada curva granulométrica nos corpos de prova exclusivos. Já os resultados na Tabela 3 são as médias dos 9 ensaios de MR por curva granulométrica após ensaio de DP.

Tabela 2: Coeficientes médios dos modelos testados de MR para 3 corpos de provas moldados por curva para as duas britas em várias granulometrias

\begin{tabular}{|c|c|c|c|c|c|c|}
\hline \multicolumn{7}{|c|}{$M R=k_{1} \cdot\left(\sigma_{3}\right)^{k 2}$} \\
\hline Variáveis & $\mathbf{C 2}$ & C3 & C4 & C5 & C6 & C7 \\
\hline k1 & 1176 & 711 & 1077 & 1178 & 631 & 919 \\
\hline k2 & 0,51 & 0,35 & 0,44 & 0,40 & 0,29 & 0,36 \\
\hline $\mathrm{R}^{2}$ & 0,91 & 0,78 & 0,84 & 0,51 & 0,55 & 0,81 \\
\hline \multicolumn{7}{|c|}{$M R=k_{1} \cdot\left(\sigma_{3}\right)^{k 2} \cdot\left(\sigma_{d}\right)^{k 3}$} \\
\hline k1 & 1283 & 786 & 1202 & 1149 & 695 & 1033 \\
\hline k2 & 0,49 & 0,45 & 0,50 & 0,53 & 0,47 & 0,44 \\
\hline k3 & 0,06 & $-0,09$ & $-0,02$ & $-0,18$ & $-0,19$ & $-0,05$ \\
\hline $\mathrm{R}^{2}$ & 0,872 & 0,522 & 0,872 & 0,443 & 0,622 & 0,834 \\
\hline
\end{tabular}

Tabela 3: Coeficientes dos MRs realizados após os ensaios de DP para as duas britas em várias granulometrias

\begin{tabular}{ccccccc}
\hline \multicolumn{7}{c}{$\mathbf{M} \boldsymbol{R}=\boldsymbol{k}_{\mathbf{1}} \cdot\left(\boldsymbol{\sigma}_{\mathbf{3}}\right)^{\mathbf{k} \mathbf{2}}$} \\
\hline Variáveis & $\mathbf{C 2}$ & $\mathbf{C 3}$ & $\mathbf{C 4}$ & $\mathbf{C 5}$ & $\mathbf{C 6}$ & $\mathbf{C 7}$ \\
k1 & 1182 & 1128 & 1580 & 1240 & 770 & 1150 \\
k2 & 0,44 & 0,50 & 0,53 & 0,40 & 0,30 & 0,39 \\
$\mathrm{R}^{2}$ & 0,43 & 0,76 & 0,81 & 0,77 & 0,80 & 0,39 \\
\hline
\end{tabular}

Ao analisar o MR, detalhados em Lima (2016), o modelo composto, confirma, para os materiais utilizados, que a tensão confinante exerce considerável influência sobre o MR, pouco influenciado pela tensão desvio. É relevante também observar que os valores de MR foram condizentes com o tipo de material analisado, brita graduada, para aplicação em base. Os menores MR foram das curvas C3 e C6 que apresentam maior quantidade de finos. Ainda observando as Tabelas 2 e 3 é possível verificar que houve aumento do MR após o material ter sido submetido a mais de $10^{5}$ solicitações de carregamento.

É possível observar nos gráficos da Figura 3 que variar a distribuição granulométrica influencia diretamente nas deformações permanentes. Na Tabela 4 ficam melhor ilustradas as variações das deformações permanentes para os 150 mil ciclos iniciais para todas as curvas granulométricas ensaiadas, sendo possível confirmar que a deformação permanente cresce com o aumento da tensão desvio e com o crescimento da relação $\sigma_{d} / \sigma_{3}$. 

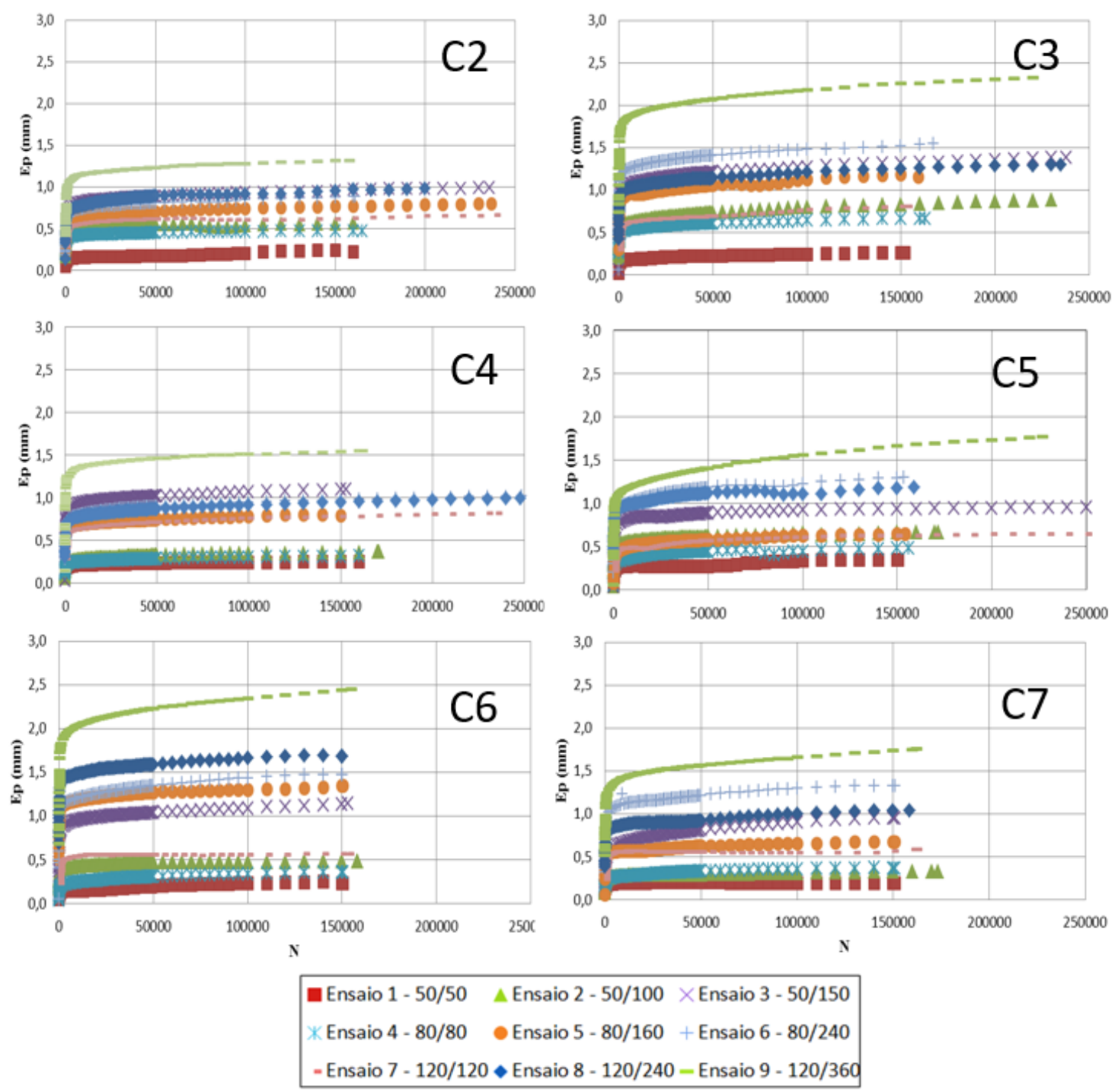

Figura 3. Resultados gráficos dos ensaios de deformações permanentes no equipamento triaxial de cargas repetidas das duas britas com diferentes granulometrias

Ainda na Tabela 4, verifica-se que quanto maior a tensão confinante maior o MR, e os MR foram menores à medida que se aumentou a relação entre as tensões, ou seja, com o aumento da tensão desvio, neste caso com exceção da C2. Vale salientar que esses MR apresentados são resultados dos modelos compostos mostrados na Tabela 2 para suas respectivas curvas.

Analisando o shakedown pelos gráficos na Figura 4, têm-se comportamentos do tipo A e B, sendo que o fato de alguns ensaios não terem atingido $10^{-7}(\times 0,001 \mathrm{~m} /$ ciclo de carga) na análise gráfica pode ser devido ao número de ciclos realizados no equipamento triaxial de cargas repetidas não ter sido suficiente. Os dois materiais apresentaram bom comportamento frente à possibilidade de surgimento de deformações permanentes que tendam ao colapso.

Um resumo dos resultados obtidos de cada uma das curvas granulométricas estudadas nesta pesquisa (ensaios de caracterização, granulometria, umidade, etc) e os parâmetros do modelo de Guimarães determinados estão na Tabela 5, onde as siglas LA, EA, $W_{o ́ t}, M E A S, D_{10}, C_{N U}$, e $C_{C}$ representam, respectivamente, os valores de Abrasão Los Angeles, equivalente de areia, umidade ótima, massa específica aparente seca máxima, diâmetro efetivo, coeficiente de não uniformidade e coeficiente de curvatura. Essas informações podem servir como preliminares para britas semelhantes. 
Tabela 4: Tabela resumo das deformações permanentes acumuladas até 150 mil ciclos de cargas e dos módulos de resiliência das duas britas com diferentes granulometrias

\begin{tabular}{|c|c|c|c|c|c|c|c|c|c|c|c|c|}
\hline \multirow{2}{*}{$\sigma_{d} / \sigma_{3}$} & \multicolumn{2}{|l|}{$\mathrm{C} 2$} & \multicolumn{2}{|l|}{ C3 } & \multicolumn{2}{|l|}{ C4 } & \multicolumn{2}{|l|}{ C5 } & \multicolumn{2}{|l|}{ C6 } & \multicolumn{2}{|l|}{ C7 } \\
\hline & DP150.000 & MR & DP150.000 & MR & DP150.000 & MR & DP150.000 & MR & DP150.000 & MR & DP150.000 & MR \\
\hline $50 / 50$ & 0,2 & 246 & 0,3 & 264 & 0,3 & 291 & 0,3 & 393 & 0,2 & 299 & 0,2 & 321 \\
\hline $100 / 50$ & 0,6 & 256 & 0,8 & 248 & 0,4 & 286 & 0,7 & 348 & 0,5 & 262 & 0,3 & 311 \\
\hline $150 / 50$ & 1,0 & 262 & 1,3 & 239 & 1,1 & 284 & 0,9 & 324 & 1,1 & 243 & 1,0 & 305 \\
\hline $80 / 80$ & 0,5 & 319 & 0,7 & 313 & 0,3 & 363 & 0,5 & 465 & 0,4 & 342 & 0,4 & 386 \\
\hline $160 / 80$ & 0,8 & 332 & 1,2 & 294 & 0,8 & 358 & 0,6 & 412 & 1,3 & 299 & 0,7 & 373 \\
\hline $240 / 80$ & 0,9 & 340 & 1,5 & 284 & 1,0 & 354 & 1,3 & 384 & 1,5 & 277 & 1,3 & 366 \\
\hline $120 / 120$ & 0,6 & 399 & 0,8 & 363 & 0,8 & 440 & 0,6 & 538 & 0,6 & 383 & 0,6 & 452 \\
\hline $240 / 120$ & 1,0 & 415 & 1,3 & 341 & 0,9 & 433 & 1,2 & 477 & 1,7 & 335 & 1,0 & 437 \\
\hline $360 / 120$ & 1,3 & 425 & 2,3 & 329 & 1,5 & 430 & 1,7 & 444 & 2,4 & 310 & 1,7 & 429 \\
\hline
\end{tabular}

Tabela 5: Tabela resumo com as informações gerais dos resultados das curvas granulométricas

\begin{tabular}{|c|c|c|c|c|c|c|c|c|c|c|c|c|c|c|c|}
\hline \multirow{2}{*}{ I } & \multirow{2}{*}{$\begin{array}{l}\text { LA } \\
(\%)\end{array}$} & \multirow{2}{*}{$\begin{array}{l}\text { EA } \\
(\%)\end{array}$} & \multirow{2}{*}{$\begin{array}{l}\text { Wót. } \\
(\%)\end{array}$} & \multirow{2}{*}{$\begin{array}{l}\text { MEAS } \\
\left(\mathrm{g} / \mathrm{cm}^{3}\right)\end{array}$} & \multicolumn{3}{|c|}{ MR } & \multirow{2}{*}{$\begin{array}{c}D_{10} \\
(\mathrm{~mm})\end{array}$} & \multirow{2}{*}{$\begin{array}{c}\mathrm{C}_{\mathrm{NU}} \\
-\end{array}$} & \multirow{2}{*}{$\begin{array}{c}\mathrm{C}_{\mathrm{c}} \\
-\end{array}$} & \multicolumn{5}{|c|}{$\varepsilon_{p}(\%)=\psi_{1} \cdot\left(\sigma_{3}\right)^{\psi_{2}} \cdot\left(\sigma_{d}\right)^{\psi_{3}} \cdot N^{\psi_{4}}$} \\
\hline & & & & & $\mathbf{k}_{1}$ & $k_{2}$ & $\mathbf{k}_{3}$ & & & & $\Psi_{1}$ & $\Psi_{2}$ & $\Psi_{3}$ & $\Psi_{4}$ & $\mathbf{R}^{2}$ \\
\hline $\mathrm{C} 2$ & 41 & 68 & 4,9 & 2,288 & 1283 & 0,49 & 0,06 & 0,17 & 47,06 & 2,38 & 0,0968 & $-0,1685$ & 0,7326 & 0,0863 & 0,895 \\
\hline C3 & 41 & 68 & 7,0 & 2,111 & 786 & 0,45 & $-0,09$ & 0,07 & 15,43 & 1,04 & 0,1276 & $-0,4085$ & 1,0500 & 0,0735 & 0,946 \\
\hline C4 & 41 & 68 & 5,4 & 2,296 & 1202 & 0,50 & $-0,02$ & 0,08 & 80,00 & 1,76 & 0,1010 & $-0,1825$ & 0,9091 & 0,0753 & 0,840 \\
\hline C5 & 43 & 71 & 5,0 & 2,223 & 1149 & 0,53 & $-0,18$ & 0,17 & 47,06 & 2,38 & 0,0868 & $-0,2801$ & 0,8929 & 0,0961 & 0,934 \\
\hline C6 & 43 & 71 & 7,5 & 2,025 & 695 & 0,47 & $-0,19$ & 0,07 & 15,43 & 1,04 & 0,1294 & $-0,0647$ & 1,1000 & 0,0735 & 0,926 \\
\hline C7 & 43 & 71 & 5,7 & 2,244 & 1033 & 0,44 & $-0,05$ & 0,08 & 80,00 & 1,76 & 0,0775 & $-0,2304$ & 1,1428 & 0,0857 & 0,951 \\
\hline
\end{tabular}

O controle da umidade ótima (Wót.) foi realizado com muita atenção, visto que a mudança de umidade pode influenciar no acréscimo de deformações permanentes. Segundo Dawson (1999), o alto teor de umidade diminui a rigidez, aumenta a susceptibilidade à deformação permanente, aumenta a permeabilidade e varia a durabilidade das camadas granulares do pavimento. Na Tabela 6 estão apresentadas as umidades de cada corpo de prova ensaiado e as umidades ótimas de cada uma das curvas granulométricas, bem como os desvios padrões relacionados.

Para este trabalho, os corpos de prova foram homogeneizados para alcançarem a umidade ótima. A verificação da umidade foi realizada por meio de cápsulas no momento da compactação e por meio do corpo de prova após ser ensaiado. É possível observar, pela Tabela 6, que a umidade após o ensaio é mais uniforme do que a umidade observada em cápsulas antes da amostra ser ensaiada. Neste caso, a variância, no geral, foi bem pequena. Além disso, não se verificou a existência de uma relação/padrão da variação da umidade das amostras antes e após o ensaio de acordo com a granulometria, nem quanto ao material em si, quando considerados os resultados de todas as amostras antes e os resultados após. Ainda assim, quando analisados os resultados referentes a cada $\mathrm{CP}$ e sua umidade ótima, comparando granulometrias semelhantes, há uma variação maior, embora pequena, para o material 2, explicada pela diferença na capacidade de absorção dos dois materiais.

Nas Tabelas 7 e 8 estão apresentadas as correlações existentes entre duas ou mais variáveis para identificar o quanto estão associadas. Os coeficientes de correlação r aqui apresentados na matriz encontrada pelo software Statistica 13.0 é uma medida de intensidade da relação entre as variáveis, mas não mede diretamente a influência que uma variável causa à outra. Na Tabela 6 estão mostradas todas as correlações obtidas entre as variáveis de deformações permanentes, 
tensão confinante, tensão desvio, índice de vazios, densidade e os coeficientes do módulo de resiliência. Os dados utilizados para Tabela 8 foram de todas as seis curvas granulométricas apresentadas neste artigo e da Brita Graduada de Chapecó investigada por Guimarães (2009). De maneira geral as dependências entre as variáveis mostradas na Tabela 8 são próximas às da Tabela 7.
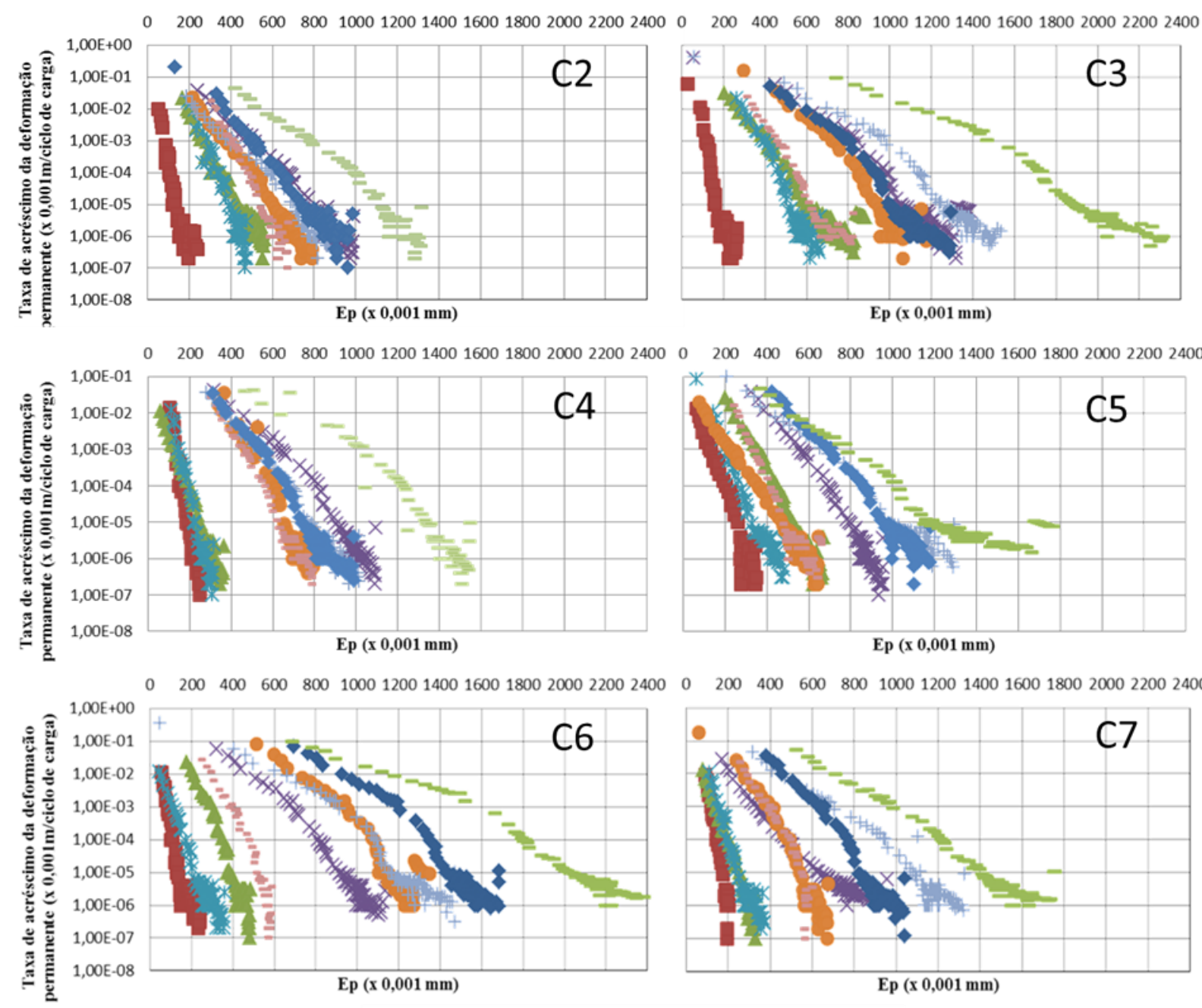

$0 \quad 200 \quad 400 \quad 600 \quad 80010001200140016001800200022002400$
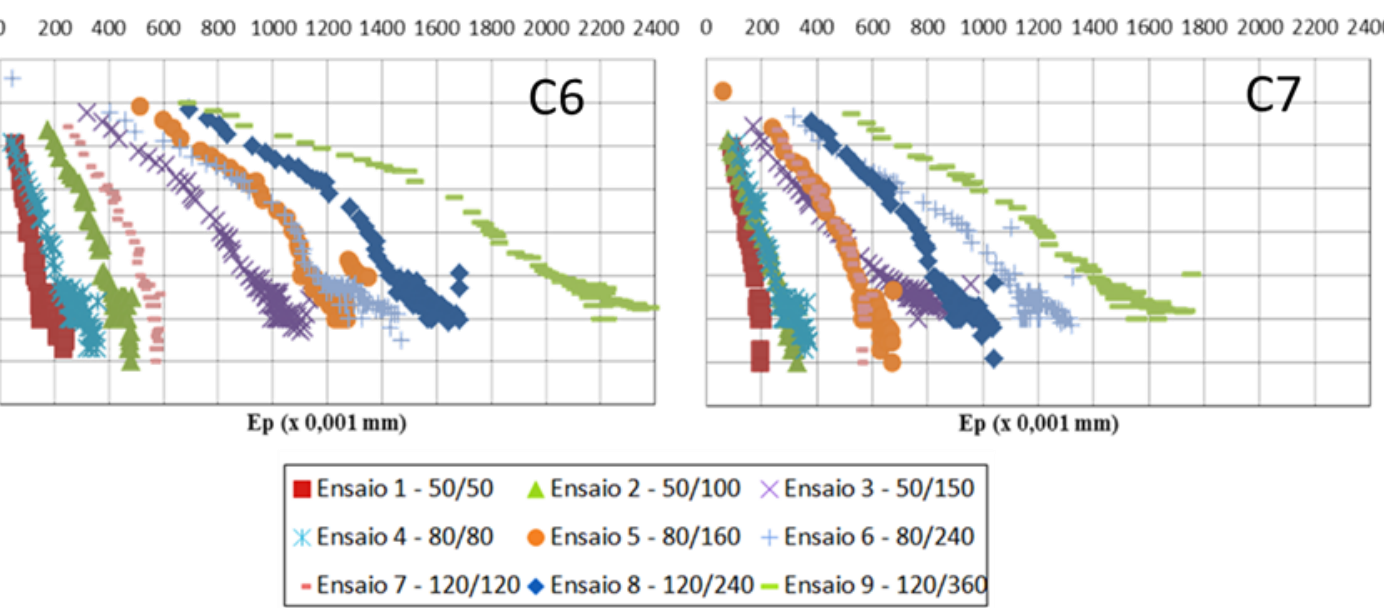

Figura 4. Pesquisa de ocorrência do Shakedown pelo modelo gráfico de Dawson e Wellner para as duas britas estudadas em várias granulometrias

Pode-se afirmar que a maior correlação com a deformação permanente foi devido à tensão desvio, e com relação positiva. Para essas duas variáveis há uma forte correlação, o que indica que a deformação permanente aumenta concomitantemente com o aumento da tensão desvio. Já a tensão confinante apresentou uma boa correlação, 0,49, relação também positiva, o que indica que está associada à mudança de níveis de deformações permanentes. A única relação entre variáveis e a deformação permanente que apresentou relação negativa, embora pequena, foi a densidade. Deve-se ao fato que as maiores densidades estão relacionadas às menores DP acumuladas. Além disso, a correlação positiva existente entre os índices de vazios e as deformações permanentes acumuladas indica que há dependência entre estas. 
Tabela 6: Umidades e desvio padrão em relação às umidades ótimas das curvas granulométricas

\begin{tabular}{|c|c|c|c|c|c|c|c|c|c|c|c|}
\hline & \multicolumn{2}{|c|}{$W_{\text {ótima }}(\%)=4,9$} & \multirow{2}{*}{$\begin{array}{c}4,9 \pm \\
\sigma\end{array}$} & & \multicolumn{2}{|c|}{$w_{\text {ótima }}(\%)=7,0$} & \multirow{2}{*}{$\begin{array}{c}7,0 \pm \\
\sigma\end{array}$} & & \multicolumn{2}{|c|}{$w_{\text {ótima }}(\%)=5,4$} & \multirow{2}{*}{$\frac{5,4 \pm}{\sigma}$} \\
\hline \multirow{11}{*}{$\mathrm{C} 2$} & $\mathbf{W}_{\text {antes }}(\%)$ & $\mathrm{w}_{\text {após }}(\%)$ & & \multirow{10}{*}{ C3 } & $\mathbf{W}_{\text {antes }}(\%)$ & $\mathbf{W}_{\text {após }}(\%)$ & & \multirow{10}{*}{ C4 } & $\mathbf{W}_{\text {antes }}(\%)$ & $\mathbf{W}_{\text {após }}(\%)$ & \\
\hline & 4,9 & 4,5 & 0,26 & & 6,9 & 6,5 & 0,30 & & 4,9 & 4,8 & 0,04 \\
\hline & 5,3 & 4,6 & 0,49 & & 6,5 & 6,5 & 0,00 & & 4,7 & 4,9 & 0,16 \\
\hline & 4,2 & 4,3 & 0,18 & & 6,6 & 6,6 & 0,10 & & 5,7 & 5,5 & 0,45 \\
\hline & 5,4 & 4,7 & 0,43 & & 6,5 & 6,5 & 0,01 & & 5,6 & 5,0 & 0,36 \\
\hline & 4,4 & 4,6 & 0,19 & & 7,2 & 6,5 & 0,40 & & 5,2 & 4,8 & 0,37 \\
\hline & 4,9 & 4,6 & 0,14 & & 5,6 & 6,4 & 0,48 & & 5,1 & 4,9 & 0,11 \\
\hline & 4,6 & 4,4 & 0,13 & & 6,6 & 6,5 & 0,09 & & 5,6 & 5,7 & 0,50 \\
\hline & 5,0 & 4,7 & 0,19 & & 6,9 & 6,9 & 0,26 & & 5,7 & 4,9 & 0,49 \\
\hline & 5,4 & 4,0 & 0,70 & & 6,1 & 6,1 & 0,23 & & 5,4 & 4,9 & 0,39 \\
\hline & \multicolumn{2}{|c|}{$W_{\text {ótima }}(\%)=5,0$} & $5,0 \pm$ & & \multicolumn{2}{|c|}{$w_{\text {ótima }}(\%)=7,5$} & $7,5 \pm$ & & \multicolumn{2}{|c|}{$W_{\text {ótima }}(\%)=5,7$} & $5,7 \pm$ \\
\hline \multirow{10}{*}{ C5 } & $\mathbf{W}_{\text {antes }}(\%)$ & $\mathbf{w}_{\text {após }}(\%)$ & $\sigma$ & \multirow{10}{*}{ C6 } & $\mathbf{w}_{\text {antes }}(\%)$ & $\mathbf{W}_{\text {após }}(\%)$ & $\sigma$ & \multirow{10}{*}{$\mathrm{C} 7$} & $\mathbf{w}_{\text {antes }}(\%)$ & $\mathbf{W}_{\text {após }}(\%)$ & $\sigma$ \\
\hline & 4,8 & 4,6 & 0,15 & & 7,3 & 6,7 & 0,39 & & 5,4 & 4,8 & 0,40 \\
\hline & 5,2 & 4,2 & 0,69 & & 7,7 & 6,9 & 0,57 & & 5,5 & 5,0 & 0,37 \\
\hline & 5,0 & 5,0 & 0,23 & & 7,2 & 6,8 & 0,26 & & 5,8 & 4,8 & 0,59 \\
\hline & 4,4 & 4,6 & 0,18 & & 7,3 & 6,8 & 0,25 & & 5,0 & 4,1 & 0,48 \\
\hline & 5,8 & 5,0 & 0,44 & & 7,3 & 6,5 & 0,39 & & 5,8 & 4,2 & 0,80 \\
\hline & 4,0 & 5,0 & 0,52 & & 7,4 & 6,9 & 0,32 & & 5,9 & 4,7 & 0,92 \\
\hline & 4,7 & 4,3 & 0,33 & & 7,3 & 7,1 & 0,39 & & 5,0 & 4,7 & 0,38 \\
\hline & 4,6 & 4,1 & 0,45 & & 7,0 & 6,8 & 0,09 & & 5,6 & 4,6 & 0,51 \\
\hline & 4,7 & 5,5 & 0,61 & & 7,4 & 7,0 & 0,21 & & 5,3 & 4,9 & 0,28 \\
\hline
\end{tabular}

$*_{\sigma}$ : Desvio Padrão

Tabela 7: Matriz de correlação entre as variáveis, das sete curvas granulométricas, obtidas para as britas deste estudo, com os dados da dissertação de Lima (2016)

\begin{tabular}{|c|c|c|c|c|c|c|c|}
\hline & $\begin{array}{c}\text { Deformação } \\
\text { permanente } \\
\text { (mm) }\end{array}$ & $\begin{array}{c}\text { Tensão } \\
\text { confinante }\end{array}$ & $\begin{array}{l}\text { Tensão } \\
\text { desvio }\end{array}$ & $\begin{array}{c}\text { Índice de } \\
\text { vazios }\end{array}$ & Densidade & \begin{tabular}{c}
\multicolumn{1}{c}{$\mathbf{k}_{1}$ do } \\
Módulo de \\
resiliência
\end{tabular} & $\begin{array}{c}\mathbf{k}_{2} \text { do } \\
\text { Módulo de } \\
\text { resiliência }\end{array}$ \\
\hline Deformação permanente (mm) & 1 & & & & & & \\
\hline Tensão confinante & 0,49 & 1 & & & & & \\
\hline Tensão desvio & 0,88 & 0,62 & 1 & & & & \\
\hline Índice de vazios & 0,27 & $-0,02$ & 0,00 & 1 & & & \\
\hline Densidade & $-0,25$ & 0,02 & 0,00 & $-0,92$ & 1 & & \\
\hline $\mathrm{k}_{1}$ do Módulo de resiliência & 0,13 & 0,11 & 0,24 & $-0,4$ & 0,48 & 1 & \\
\hline $\mathrm{k}_{2}$ do Módulo de resiliência & 0,13 & 0,10 & 0,14 & $-0,22$ & 0,41 & 0,87 & 1 \\
\hline
\end{tabular}

Tabela 8: Matriz de correlação entre as possíveis correlações das informações obtidas com os dados dos trabalhos Lima e Motta (2015a, 2015b, 2016) e Guimarães (2009)

\begin{tabular}{|c|c|c|c|c|c|}
\hline & $\begin{array}{c}\text { Deformação } \\
\text { permanente }(\mathrm{mm})\end{array}$ & $\begin{array}{c}\text { Tensão } \\
\text { confinante }\end{array}$ & $\begin{array}{l}\text { Tensão } \\
\text { desvio }\end{array}$ & $\begin{array}{l}\mathbf{k}_{1} \text { do Módulo } \\
\text { de resiliência }\end{array}$ & $\begin{array}{l}\mathrm{k}_{2} \text { do Módulo } \\
\text { de resiliência }\end{array}$ \\
\hline Deformação permanente (mm) & 1 & & & & \\
\hline Tensão confinante & 0,40 & 1 & & & \\
\hline Tensão desvio & 0,89 & 0,55 & 1 & & \\
\hline k do Módulo de resiliência & 0,16 & 0,06 & 0,25 & 1 & \\
\hline k2 do Módulo de resiliência & 0,18 & 0,02 & 0,16 & 0,88 & 1 \\
\hline
\end{tabular}

Observando ainda as Tabelas 7 e 8, a correlação entre as deformações permanentes acumuladas e o coeficiente $\mathrm{k} 2$, do MR simples, obtido pela tensão confinante variaram muito pouco, mas de forma positiva. Houve considerável variação da DP para k2 e k1 praticamente inalterados entre um pequeno intervalo para cada. Da mesma forma que k2, k1 apresenta uma relação 
positiva, porém diferente das demais variáveis, essas correlações entre as deformações permanentes acumuladas e o MR (k1 e k2) não foram tão fortes, o que tem sentido em razão da dispersão dos pontos.

\section{CONCLUSÕES}

Os ensaios de deformação permanente das diferentes curvas granulométricas para dois materiais granito-gnaisse de duas pedreiras do Rio de Janeiro apresentaram comportamentos semelhantes quanto ao surgimento das deformações permanentes de acordo com os níveis de tensões aplicados e suas relações. As deformações permanentes acumuladas são maiores para razões de tensões maiores, comportamento verificado para outros tipos de materiais.

Para brita graduada granito-gnaisse, os valores apresentados foram adequados para aplicação em camadas de base e sub-base, embora melhores para algumas composições granulométricas do que para outras. De forma geral, apresentaram resultados baixos de deformações permanentes acumuladas, estimando-se pequena contribuição da camada se utilizada espessura de $20 \mathrm{~cm}$ total deste material. 0 valor admissível de 12,5 mm de ATR total é geralmente considerado em projetos de rodovias de alto volume de tráfego. A combinação dos resultados reflete a importância da realização de ensaios triaxiais de cargas repetidas para verificação da capacidade mecânica do material a ser empregado nas camadas do pavimento e assim poder prever o surgimento de deformação permanente.

Pôde-se concluir que os valores dos índices de vazios e densidades dos dois materiais britados apresentaram uma leve tendência de correlação com a DP, apesar da baixa correlação, pois quanto menores os índices de vazios menores as deformações permanentes, enquanto as densidades foram inversamente proporcionais às deformações permanentes.

A tensão confinante e a tensão desvio apresentaram boa correlação com relação à deformação permanente, sendo que a tensão desvio apresentou a maior das correlações, o que condiz com os parâmetros encontrados por Guimarães (2009), já que $\Psi_{3}$ (parâmetro de deformabilidade referente à tensão desvio) apresentou maior influência para todas as curvas. Quanto à umidade, não houve influência e correlação com os demais parâmetros, pois os ensaios foram realizados visando a homogeneidade ótima para o material em determinada distribuição granulométrica.

As duas formas para encontrar o módulo de resiliência descritas nesta pesquisa, ensaios de módulo de resiliência tradicionais em corpos de provas unicamente moldados para esse propósito, três por curva, e a realização de módulo de resiliência em corpos de provas já submetidos ao ensaio de deformação permanente, permitiram verificar que os módulos de resiliência aumentaram após o longo período de aplicação de cargas (mínimo de 150 mil). Essa análise indicou que o material pode enrijecer e que em campo esse comportamento pode ocorrer quando houver o movimento repetido das cargas impostas pelo tráfego. Maiores detalhes podem ser consultados na dissertação de Lima (2016).

Vale ainda salientar que os parâmetros de Guimarães (2009) para as britas graduadas com as distribuições granulométricas aqui investigadas irão contribuir para o banco de dados para futura utilização desse modelo no Método de Dimensionamento Mecanístico-Empírico de Pavimentos Asfálticos Brasileiro - SisPavBR.

\section{REFERÊNCIAS}

Alessandri, A., A. Di Febbraro, A. Ferrara and E. Punta (1998). Optimal control of freeways via speed signalling and ramp metering. Control Engineering Practice, v. 6, n. 6, p. 771-780. DOI: 10.1016/S0967-0661(98)00083-5. 
Barksdale, R. D (1972). Laboratory Evaluation of Rutting in Base Course Materials. In: INTERNATIONAL CONFERENCE ON STRUCTURAL DESIGN OF ASPHALT PAVEMENTS, 3., 1972. Proceedings..., London, pp. 161-174.

Cerni, S., Cardone, F., Virgili, A., et al. (2012). Characterization of permanent deformation behaviour of unbound granular materials under repeated triaxial loading. Construction and Building Materials, n. 28, p. 79-87.

DOI: 10.1016/j.conbuildmat.2011.07.066.

Dawson, A. R. (1999). Implications of granular material characteristics on the response of different pavement constructions. In: SYMPOSIUM ON MODELLING \& ADVANCED TESTING FOR UNBOUND GRANULAR MATERIALS, 1999. Proceeding... Balkema. p. 221-226.

Dawson A. R. e Wellner F. (1999). Plastic behavior of granular materials. Final Report ARC Project 933. University of Nottingham.

Guimarães, A. C. R (2009). Um método mecanítico-empírico para a previsão da deformação permanente em solos tropicais constituintes de pavimentos. Tese (Doutorado) - Programa de Engenharia Civil da COPPE/UFRJ. Rio de Janeiro, RJ.

Lima, C. D. A. e Motta, L. M. G. (2015a). Influência da variação granulométrica de brita graduada simples na deformação permanente. In: ANPET - CONGRESSO DE PESQUISA E ENSINO EM TRANSPORTES, 29., 2015. Anais... Ouro Preto, Brasil.

Lima, C. D. A. e Motta, L. M. G. (2015b). Estudo da deformação permanente de uma brita graduada. In: CONGRESSO IBEROLATINO AMERICANO DO ASFALTO, 18., 2015. Anais... Bariloche, Argentina.

Lima, C. D. A. (2016). Estudo da deformação permanente de duas britas graduadas para uso em camadas de pavimentos. Dissertação (Mestrado) - Programa de Engenharia Civil da COPPE/UFRJ. Rio de Janeiro, RJ.

Lima, C. D. A. e Motta, L. M. G. (2016). Study of Permanent Deformation and Granulometric Distribution of Graded Crushed Stone Pavement Material. Advances in Transportation Geotechnics, v. 143, p. 854-861. Guimarães, Portugal.

DOI: 10.1016/j.proeng.2016.06.141.

Marangon, M. e Motta, L. M. G. (2006). Estudo da deformação permanente de alguns solos argilosos lateríticos visando o uso em pavimentos de baixo volume de tráfego. In: II SIMPÓSIO BRASILEIRO DE JOVENS GEOTÉCNICOS, 2., 2006. Anais... Nova Friburgo/RJ : ABMS ; UERJ.

Rede Temática de Asfalto (2010). Manual de execução de trechos monitorados: rede temática de tecnologia em asfalto. Rio de Janeiro, RJ: Petrobras.

Werkmeister, S. (2003). Permanent deformation behavior of unbound granular materials in pavement constructions. Tese (Doutorado) - Universidade Técnica de Dresden. Dresden.

Werkmeister, S.; Dawson, A. R.; Wellner, F. (2004). Pavement design for unbound granular materials. Journal of Transportation Engineering, v. 130, n. 5, Sep./Oct. 